\title{
Strength of the null singularity inside black holes
}

\author{
Lior M. Burko \\ Theoretical Astrophysics, California Institute of Technology, Pasadena, California 91125
}

(Received 3 May 1999; published 26 October 1999)

\begin{abstract}
We study analytically the Cauchy horizon singularity inside spherically symmetric charged black holes, coupled to a spherical, self-gravitating, minimally coupled, massless scalar field. We show that all causal geodesics terminate at the Cauchy horizon at a null singularity, which is weak according to the Tipler classification. The singularity is also deformationally weak in the sense of Ori. Our results are valid at arbitrary points along the null singularity, in particular at late retarded times, when nonlinear effects are crucial. [S0556-2821(99)04920-6]

PACS number(s): 04.70.Bw, 04.20.Dw
\end{abstract}

\section{INTRODUCTION}

The issue of spacetime singularities - which are known to inevitably occur inside black holes under very plausible assumptions [1] - is an intriguing puzzle of physics. The laws of physics, as we presently understand them (e.g., classical general relativity), are presumably invalid at singularities. Instead, some other theories (e.g., quantum gravity), as yet unknown, are expected to take over from general relativity and control the spacetime structure. The general relativistic predictions are nevertheless of the greatest importance, as they reveal the spacetime structure under extreme conditions in the strong-field regime. Of particular interest is the possibility that there are two distinct ways in which general relativity can fail at different types of singularities: for one type of singularity the failure is through infinite destructive effects on physical objects, whereas for the other type the failure is through the breakdown of predictability.

Until recently, the only known generic singularity in general relativity was the Belinsky-Khalatnikov-Lifshitz (BKL) singularity [2]. According to the BKL picture, spacetime develops a succession of Kasner epochs in which the axes of contraction and expansion change directions chaotically. This succession ends at unbounded oscillations at a spacelike singularity, which is unavoidably destructive for any physical object-a strong singularity. In the last several years, however, evidence has been accumulating that the BKL singularity is not the only type of singularity which may evolve in general relativity from generic initial data.

The new type of singularity forms at the Cauchy horizon $(\mathrm{CH})$ of spinning or charged black holes. (For a recent review see Ref. [3].) The features of this singularity are markedly different from those of the BKL singularity: (i) It is null (rather than spacelike), (ii) it is weak (according to Tipler's classification [4]), specifically, the tidal deformations which an extended physical object suffers upon approaching the singularity are bounded. In the case of a spinning black hole, the evidence for the null and weak singularity has emerged from analytical perturbative [5,6] and nonperturbative [7] analyses. In addition, the local existence and genericity of a null and weak singularity in solutions of the vacuum Einstein equations was demonstrated in Ref. [8]. This was more recently demonstrated also in the framework of planesymmetric spacetimes in Ref. [9]. For the toy model of a spherical charged black hole, the main features of the $\mathrm{CH}$ singularity were first found analytically for simplified models based on null fluids [10-12], and later confirmed numerically for a model with a self-gravitating scalar field [13,14]. Expressions for the divergence rate of the blueshift factors for that model, which are valid everywhere along the $\mathrm{CH}$, were found analytically in Ref. [15]. Those expressions are exact on the $\mathrm{CH}$ as functions of retarded time. However, they are only asymptotic expressions as functions of advanced time (see below).

The strength of the null singularity is of crucial importance for the question of the hypothetical possibility of hyperspace travel through the $\mathrm{CH}$ of black holes. A necessary condition for this possibility to be realized is that physical objects would traverse the $\mathrm{CH}$ peacefully. Because the $\mathrm{CH}$ is known to be a curvature singularity, it is necessary that the singularity would be weak according to the Tipler classification of singularity strengths. For the toy model of a spherical charged black hole, which we shall study here, the properties of the $\mathrm{CH}$ singularity which have been found in Refs. [1115] are all consistent with the picture of a Tipler weak singularity. However, the weakness of the singularity was demonstrated only for the simplified Ori model [12] and at asymptotically early times for spinning black holes [5], where there are still no strong nonlinear effects, such as focusing of the null generators of the $\mathrm{CH}$, which are crucial at later times. In the context of spherical charged black holes and a self-gravitating scalar field, several important features of the spacetime structure have been found in fully nonlinear numerical simulations. Specifically, it was shown that for any point along the $\mathrm{CH}$ singularity there existed coordinates for which the metric coefficients were finite and the metric determinant was nondegenerate in an open neighborhood to the past $[13,14]$. However, despite previous claims $[5,14,16]$, this still does not guarantee that the singularity is weak in the Tipler sense [17].

It is the purpose of this paper to present an analytical demonstration of the weakness of the singularity for the model of a spherical charged black hole with a selfgravitating, minimally coupled, massless, real scalar field. Our results are valid at arbitrary points along the $\mathrm{CH}$ singularity, in particular at late times, where strong nonlinear effects (focusing of the null generators of the $\mathrm{CH}$ and growth of the blueshift factors) are crucial. In fact, our results are 
valid everywhere along the $\mathrm{CH}$ singularity, down to the event where the generators of the $\mathrm{CH}$ are completely focused, and the singularity becomes spacelike and Tipler strong [18]. We emphasize that although our discussion here is analytical, we do make assumptions which are based on results obtained by numerical simulations.

\section{STRENGTH OF THE SINGULARITY}

We write the general spherically symmetric line element in the form

$$
d s^{2}=-f(u, \mathrm{v}) d u d \mathbf{v}+r^{2}(u, \mathrm{v}) d \Omega^{2},
$$

where $d \Omega^{2}=d \vartheta^{2}+\sin ^{2} \vartheta d \varphi^{2}$ is the line element on the unit two sphere. The coordinates $u, \mathrm{~V}$ are any outgoing and ingoing null coordinates, correspondingly. (Below, we shall specialize to a specific choice of gauge, and define a particular choice of an ingoing null coordinate.) We consider the class of scalar field perturbations which is inherent to any gravitational collapse process. These are the perturbations which result from the evolution of nonvanishing multipole moments during the collapse. When these perturbations propagate outwards, they are partially reflected off the spacetime curvature and captured by the black hole. This process results in a scalar field, which at late advanced times decays along the event horizon according to an inverse power of advanced time. Specifically, we assume that the scalar field behaves along the event horizon at late times according to $\Phi^{\mathrm{EH}} \propto\left(\kappa \mathrm{V}_{e}\right)^{-n}[19-21]$, where $n$ is a positive integer which is related to the multipole moment under consideration. (We do not consider, however, other possible sources of perturbations [22].) By $\mathrm{v}_{e}$ we denote the usual advanced time in the Eddington gauge, and $r_{ \pm}, \kappa$ are the outer and inner horizons and the surface gravity of the latter, respectively, for a Reissner-Nordström black hole having the same external parameters as the black hole we consider has at late times. We define the dimensionless ingoing Kruskal-like coordinate by $V \equiv-\exp \left(-\kappa \mathrm{V}_{e}\right)$. In the Kruskal gauge we denote the metric function $g_{u V}$ by $-F / 2$. For this model, it can be shown analytically that at arbitrary points along the $\mathrm{CH}$ the following relations are satisfied, to the leading orders in $[-\ln (-V)]^{-1}$ [15]

$$
\begin{aligned}
r_{, V}= & \frac{\left(n r_{-} A\right)^{2}}{r V}[-\ln (-V)]^{-2 n-2}\left\{1+b_{1}[-\ln (-V)]^{-1}\right. \\
& \left.+b_{2}[-\ln (-V)]^{-2}+O[-\ln (-V)]^{-3}\right\} \\
\Phi_{, V}= & \frac{\left(n r_{-} A\right)}{r V}[-\ln (-V)]^{-n-1}\left\{1+c_{1}[-\ln (-V)]^{-1}\right. \\
& \left.+c_{2}[-\ln (-V)]^{-2}+O[-\ln (-V)]^{-3}\right\} .
\end{aligned}
$$

Here, $A=\left[r_{+} /\left(2 r_{-}\right)\right]\left(r_{+} / r_{-}+r_{-} / r_{+}\right)$, and the expansion coefficients $b_{i}$ and $c_{i}$ are functions of retarded time only. Note that in the limit $V \rightarrow 0$ these are exact expressions as functions of retarded time. That is, to the leading order in $[-\ln (-V)]^{-1}$ there is implicit dependence on retarded time through $r=r(u)$, and along the $\mathrm{CH}$ singularity both $r_{, V}$ and
$\Phi_{, V}$ are exactly inversely proportional to $r(u)$, in the following sense. Consider two outgoing null rays, and let one ray be at $u=u_{1}$, say, and the other at $u=u_{2}$. The ratios $r_{, V}(2) / r_{, V}(1)$ and $\Phi_{, V}(2) / \Phi_{, V}(1)$ approach $r\left(u_{1}\right) / r\left(u_{2}\right)$ as $V \rightarrow 0$. Taking now $u_{1}$ to be in the asymptotically early parts of the $\mathrm{CH}$, where $r\left(u_{1}\right) \approx r_{-}$, we find that both $r_{, V}$ and $\Phi_{, V}$ are inversely proportional to $r(u)$. As $r(u)$ is monotonically decreasing as a function of retarded time along the $\mathrm{CH}$, we find that $r_{, V}$ and $\Phi_{, V}$ grow monotonically along the $\mathrm{CH}$. This growth is a nonlinear effect which indicates the strengthening of the singularity along the $\mathrm{CH}$ (although the singularity is still weak according to the Tipler classification; see below).

All the nonzero components of the Riemann-Christoffel curvature tensor $R_{\mu \nu \rho \sigma}$ are given completely in terms of the divergent blueshift factors $r_{, V}, \Phi_{, V}$, and the finite quantities $r, r_{, u}, \Phi_{, u}$, and $F$. Interestingly, $R_{\mu \nu \rho \sigma}$ does not depend on gradients of $F$. This can be understood from the following consideration. The tensor $R_{\mu \nu \rho \sigma}$ can be written as the sum of the Weyl tensor, and another tensor which is built from the Ricci and the metric tensors (but not involving their derivatives). In spherical symmetry the Weyl tensor is given completely in terms of the mass function, which is defined by $r_{, \mu} r^{\mu}=1-2 M(u, \mathrm{v}) / r+q^{2} / r^{2}, q$ being the charge of the black hole. In Kruskal-like coordinates the mass function $M(u, V)=(r / 2)\left(1+4 r_{, u} r_{, V} / F\right)+q^{2} /(2 r)$, which depends only on $r, r_{, V}, r_{, u}$, and $F$. (The divergence of the mass function at the singularity, and consequently also the divergence of curvature, is evident from the divergence of $r_{, V}$ and the finiteness of $r, r_{, u}$, and $F$.) The Ricci tensor $R_{\mu \nu}$ $=2 \Phi_{, \mu} \Phi_{, \nu}$, and consequently $R_{\mu \nu \rho \sigma}$ is independent of gradients of $F$.

We find that the components of $R_{\mu \nu \rho \sigma}$ which have the strongest divergence near the $\mathrm{CH}$ are $R_{V \varphi V \varphi}$ and $R_{V \vartheta V \vartheta}$. (In the Appendix we list all the nonzero independent components of the Riemann-Christoffel tensor.) It can be readily shown that

$$
R_{V \vartheta V \vartheta}=-r^{2}\left(\Phi_{, V}\right)^{2}=\sin ^{-2} \vartheta R_{V \varphi V \varphi} .
$$

We denote these two components schematically and collectively by $\mathcal{R}$. We find that, to the leading orders in $V$ and in $[-\ln (-V)]^{-1}$,

$$
\mathcal{R}(V) \propto V^{-2}[-\ln (-V)]^{-2 n-2} .
$$

(The other divergent components of $R_{\mu \nu \rho \sigma}$, i.e., $R_{u V u V}, R_{\vartheta \varphi \vartheta \varphi}, R_{u \vartheta V \vartheta}$, and $R_{u \varphi V \varphi}$ are proportional to the leading order in $V$ to $V^{-1}$ times a logarithmic factor.) Because both metric functions $r$ and $F$ have finite values at the $\mathrm{CH}$ (which is known from the numerical simulations of Refs. $[13,14])$, it is easy to show that the dependence of $\mathcal{R}(V)$ on $V$ does not change when we transform to a parallelpropagated frame.

We next find $V(\tau)$ as a function of affine parameter (proper time) $\tau$ along a general null (timelike) geodesic. For general causal geodesics, the geodesic equations are

$$
\dot{u} \dot{\mathrm{V}}=\left(m r^{2} \dot{\Omega}^{2}-p\right),
$$




$$
\begin{aligned}
& \ddot{\mathrm{v}}+\left(f_{, \mathrm{v}} / f\right) \dot{\mathrm{v}}^{2}+2 m r_{, u}(f \dot{u} \dot{\mathrm{v}}+p) /(r f)=0, \\
& \ddot{u}+\left(f_{, u} / f\right) \dot{u}^{2}+2 m r_{, \mathrm{v}}(f \dot{u} \dot{\mathrm{v}}+p) /(r f)=0 .
\end{aligned}
$$

Here, $m=0(1)$ for radial (nonradial) geodesics, and $p$ $=0(-1)$ for null (timelike) geodesics. A dot denotes differentiation with respect to affine parameter (proper time), and $\dot{\Omega}^{2}=\dot{\vartheta}^{2}+\sin ^{2} \vartheta \dot{\varphi}^{2}$. The geodesic equations can be solved to the leading order in $[\ln (-V)]^{-1}$ for all causal geodesics. This is done by using the field equation [14]

$$
F_{, V} / F=r_{, V V} / r_{, V}+r\left(\Phi_{, V}\right)^{2} / r_{, V}
$$

to find $F_{, V} / F$ explicitly. Substituting Eqs. (2) and (3) in Eq. (9) we find

$$
\begin{aligned}
(\ln F)_{, V}= & {\left[\ln \left(-r_{, V}\right)\right]_{, V}+\frac{1}{V}+\left(2 c_{1}-b_{1}\right) \frac{1}{V}[-\ln (-V)]^{-1} } \\
& +\left(b_{1}^{2}-b_{2}-2 b_{1} c_{1}+c_{1}^{2}+2 c_{2}\right) \frac{1}{V}[-\ln (-V)]^{-2} \\
& +O\left\{\frac{1}{V}[-\ln (-V)]^{-3}\right\}
\end{aligned}
$$

Integration yields

$$
\begin{aligned}
\ln F= & \ln \widetilde{F}_{0}+\ln \left(V r_{, V}\right)-\left(2 c_{1}-b_{1}\right)\{\ln [-\ln (-V)]\} \\
& +\left(b_{1}^{2}-b_{2}-2 b_{1} c_{1}+c_{1}^{2}+2 c_{2}\right)[-\ln (-V)]^{-1} \\
& +O\left\{[-\ln (-V)]^{-2}\right\} .
\end{aligned}
$$

Here, $\ln \widetilde{F}_{0}$ is an integration constant, which can be a function of $u$. Exponentiating both sides, and substituting Eq. (2) for $r, V$ we find

$$
\begin{aligned}
F= & \widetilde{F}_{0} \frac{\left(n r_{-} A\right)^{2}}{r}[-\ln (-V)]^{-2 n-2+b_{1}-2 c_{1}} \\
& \times\left\{1+b_{1}[-\ln (-V)]^{-1}+O[-\ln (-V)]^{-2}\right\} \\
& \times \exp \left\{\left(b_{1}^{2}-b_{2}-2 b_{1} c_{1}+c_{1}^{2}+2 c_{2}\right)[-\ln (-V)]^{-1}\right. \\
& \left.+O[-\ln (-V)]^{-2}\right\} .
\end{aligned}
$$

From the numerical results of Refs. $[13,14]$ it is known that as $V \rightarrow 0, F$ approaches a finite value. Consequently, in order to have consistency with the numerical results we require that $b_{1}-2 c_{1}=2 n+2$, which implies that

$$
F=F_{0}(u)\left\{1+B[-\ln (-V)]^{-1}+O[-\ln (-V)]^{-2}\right\},
$$

where $\quad B=(2 n+3) b_{1}-b_{2}+c_{1}^{2}+2 c_{2}, \quad$ and $\quad F_{0}(u)$ $=\widetilde{F}_{0}\left(n r_{-} A\right)^{2} / r$. For the logarithmic derivative of $F$ we find, to the leading order in $[-\ln (-V)]^{-1}$, that

$$
F_{, V} / F=B \frac{1}{V}[-\ln (-V)]^{-2} \text {. }
$$

(Higher order terms in $[-\ln (-V)]^{-1}$ are functions of retarded time.) We note that only $b_{1}$ and $c_{1}$ are constrained. The coefficients of higher-order terms in $[-\ln (-V)]^{-1}$ are immaterial near the $\mathrm{CH}$ for our determination of the strength of the singularity. Note that $F \rightarrow F_{0}(u)$ as $V \rightarrow 0$, and that $F$ is not analytic in $V$. In fact, this is an important property of the $\mathrm{CH}$ singularity: In a Kruskal-like gauge the metric functions $r$ and $F$ are finite at the singularity, but their gradients in the outgoing direction diverge. The finiteness of $r$ and $F$ at the $\mathrm{CH}$ also implies that the metric determinant is nondegenerate. This expression for $F$ is similar to the behavior of the $g_{u V}$ metric function found for the simplified Ori model [12]. We stress that although this expression for $F$ is exactly valid everywhere along the $\mathrm{CH}$ singularity, it still does not allow us to find the variation of $F$ with retarded time, as we do not know the form of $F_{0}(u)$ or $r(u)$ along the $\mathrm{CH}$. We note that near the $\mathrm{CH}$ the metric function $F$ is monotonic in $V$. This result is in accord with the numerical results of Ref. [14]. (Notice, however, the disagreement with the numerical results of Ref. [16]. It is reasonable to expect the behavior of the metric functions near the $\mathrm{CH}$ to be similar for both cases of real and complex scalar fields. The lower panels of Figs. 3 of Ref. [16] imply, however, a nonmonotonic behavior of $F$. That kind of behavior can be obtained from a numerical code with a specific choice of parameters if the latter is far from convergence near the $\mathrm{CH}$.)

Let us consider first radial geodesics. (The case of nonradial geodesics will be treated next.) In the null case $(m=0$ and $p=0$ ) it is easy to solve the geodesic equations (6)-(8). For outgoing geodesics one readily finds that the solution is $u=$ const and $\dot{V}=$ const $/ F$. The metric function $F$ can be expanded in $[-\ln (-V)]^{-1}$, despite its nonanalyticity in $V$. To the leading orders in $[-\ln (-V)]^{-1}$ we find that

$$
F=F_{0}(u)\left\{1+B[-\ln (-V)]^{-1}\right\},
$$

such that

$$
\dot{V}=\frac{\text { const }}{F_{0}}\left\{1-B[-\ln (-V)]^{-1}\right\} .
$$

The solution for $V(\tau)$ is then given asymptotically close to the $\mathrm{CH}$, to the leading orders in $[-\ln (-V)]^{-1}$ by

$$
V(\tau)=\tau\left\{1-B[-\ln (-\tau)]^{-1}\right\} .
$$

(Recall that the affine parameter is given up to a linear transformation.) To the leading order in $[-\ln (-\tau)]^{-1}$ we can thus approximate $V(\tau) \approx \tau$. Note that although asymptotically $V(\tau)$ and $\dot{V}(\tau)$ behave as $\tau$ and $\dot{\tau} \equiv 1$, correspondingly, $\ddot{V}(\tau)$ behaves very differently from $\ddot{\tau} \equiv 0$. In fact, $\ddot{V}(\tau)$ diverges as $\tau \rightarrow 0$. Therefore, one can approximate $V(\tau) \approx \tau$ only if one is interested in $V(\tau)$ itself, or at the most in $\dot{V}(\tau)$. This approximation is invalid for $\ddot{V}(\tau)$ or higher derivatives. For radial timelike geodesics $(m=0$ and $p=-1)$ one uses the finiteness of $F_{, u}$ to find approximately that again $\dot{V}$ $\approx$ const $F$, and consequently one finds the same result for $V(\tau)$. In the case of nonradial geodesics $(m=1)$ one can consider a specific value of the retarded time at which the 
geodesic hits the $\mathrm{CH}$ singularity. Than, $r, r_{, u}$, and $F$ can be approximated by their values at the singularity. When this is done, the equations for null nonradial $(m=1$ and $p=0)$ geodesics become inhomogeneous linear equations. The corresponding homogeneous equations are nothing but the equations for the radial geodesics, which we already solved. Particular solutions for the inhomogeneous equations are easy to generate, and one finds that again $V(\tau)$ is given asymptotically as before. The last case is the case of nonradial timelike $(m=1$ and $p=-1)$ geodesics. In this case the geodesic equation becomes (under similar assumptions) an inhomogeneous non-linear equation. Although this equation is hard to solve directly, it can be checked that the same leading order proportionality of $V(\tau)$ and $\tau$ is the solution also for this case. We thus find that for all causal geodesics, to the leading order in $[-\ln (-\tau)]^{-1}, V(\tau)$ is proportional to $\tau$.

We next reexpress $\mathcal{R}$ as a function of affine parameter (proper time) along radial or nonradial null (timelike) geodesics. To the leading order in $[-\ln (-\tau)]^{-1}$ we find that in a parallel-propagated frame

$$
\mathcal{R}(\tau) \propto \tau^{-2}[-\ln (-\tau)]^{-2 n-2} .
$$

A necessary condition for a singularity to be strong in the Tipler sense is given by the following theorem [23]: For null (timelike) geodesics, if the singularity is strong in the Tipler sense, then for at least one component of the RiemannChristoffel curvature tensor in a parallel-propagated frame, the twice integrated component with respect to affine parameter (proper time) does not converge at the singularity. Specifically, the necessary condition for the singularity to be Tipler strong is that

$$
\mathcal{I}(\tau)=\int^{\tau} d \tau^{\prime} \int^{\tau^{\prime}} d \tau^{\prime \prime}\left|\mathcal{R}\left(\tau^{\prime \prime}\right)\right|
$$

does not converge as $\tau \rightarrow 0$. It can be readily shown that when $\mathcal{R}(\tau)$ is integrated twice with respect to $\tau, \mathcal{I}(\tau)$ converges in the limit $\tau \rightarrow 0$. Consequently, we find that a necessary condition for any causal geodesic to terminate at a Tipler strong singularity is not satisfied. Hence, all causal geodesics terminate at a Tipler weak singularity, namely, the singularity is Tipler weak. The physical content of this result is that the volume element of physical objects remains bounded at the singularity. We emphasize that this result is valid everywhere along the singularity, in particular at late retarded times where the nonlinear effects (focusing of the generators of the $\mathrm{CH}$ and the growth of the blueshift factors) are crucial.

\section{CONCLUDING REMARKS}

Recently, Ori suggested to define a deformationally strong singularity in the following way. Let $\lambda(\tau)$ be a timelike geodesic with proper time $\tau$ along it. The geodesic $\lambda(\tau)$ terminates at a deformationally-strong singularity at $\tau=0$ if at least one of the following two conditions holds: (i) $\lambda(\tau)$ terminates at a Tipler strong singularity or (ii) there exists a
Jacobi field $\mathbf{J}(\tau)$ for which at least one parallel-propagated tetrad component is unbounded at the limit $\tau \rightarrow 0$ [24]. This definition is more physically motivated than Tipler's definition, because it classifies a singularity as strong not only when the volume element vanishes, but also when the volume element diverges to infinity, or there is infinite compression in one direction, and infinite stretching in a different direction, such that the volume element remains bounded. In fact, it can be shown that the failure of the necessary condition for the singularity to be Tipler strong implies not only the boundedness of the volume element, but also the boundedness of the Jacobi fields themselves [24], such that objects are not expected to be destroyed also because of distortions which preserve the volume element or divergence to infinity of the volume element. Consequently, the singularity we are studying here is weak also in the sense of Ori (deformationally weak). (Ori's definition does not include null geodesics. However, extended physical objects move along timelike geodesics, such that this deficiency does not restrict our discussion. It is conceivable that both Ori's definition and theorem for the necessary condition for the singularity to be Ori strong can be generalized to all causal geodesics.)

We note that according to the Królak classification of singularities [25] this is a strong singularity. Specifically, if we integrate over the divergent components of the RiemannChristoffel tensor only once, the integral does not converge on the singularity. This means that the expansion diverges (negatively) on the singularity (Królak strong), but still the volume element (and the distortion in general) remains finite (Tipler and Ori weak). One might be worried that even if spacetime were classically extendible beyond the $\mathrm{CH}$, this infinite negative expansion would inevitably result in unavoidable destruction of any extended physical object subsequent to its traversing of the $\mathrm{CH}$ [26]. Of course, any classical extension of geometry beyond the $\mathrm{CH}$ is not unique. We can, however, consider an extension with a continuous $\left(C^{0}\right)$ metric and a unique $C^{1}$ timelike geodesic, and assume that the object follows this geodesic [27]. Any extension of classical geometry beyond the $\mathrm{CH}$ (which can be modeled as a thin layer wherein the geometry is inherently quantum) requires an infinite flux of negative energy traveling along the contracting $\mathrm{CH}$. This negative energy flux may then act to regularize the expansion, such that the deformation rate of physical objects beyond the $\mathrm{CH}$ would be bounded [27]. (The infinite expansion is likely not to destroy physical objects up to the $\mathrm{CH}$ [27], in contrast with Ref. [26].) Indeed, a simplified two-dimensional quantum model shows an infinite ingoing flux of negative energy along the $\mathrm{CH}[28,29]$. More recent semiclassical toy models of a quantum field on a mass-inflation background are not inconsistent with this picture [30,31]. One should not take these quantum results too seriously, however, because in these models the semiclassical contributions are dominated by the regime where curvature is Planckian, such that the semiclassical approximation is not expected to be valid anymore. Instead, a full quantum theory of gravity is of need. Of course, in the absence of a valid theory of quantum gravity it is difficult to make predictions on the detailed interaction of the thin layer of the $\mathrm{CH}$ with physical objects, but the evidence we currently have 
do not preclude the possibility of objects traversing the $\mathrm{CH}$ singularity peacefully.

\section{ACKNOWLEDGMENTS}

I have benefited from useful discussions with Patrick Brady and Amos Ori. This work was supported by NSF grant AST-9731698.

\section{APPENDIX}

The independent components of the Riemann-Christoffel curvature tensor which do not vanish identically are

$$
\begin{aligned}
R_{u \mathrm{v} u \mathrm{v}} & =-\frac{1}{4} \frac{f}{r^{2}}\left[4 r_{, u} r_{, \mathrm{v}}+f\left(1-2 \frac{q^{2}}{r^{2}}\right)\right]+f \Phi_{, u} \Phi_{, \mathrm{v}}, \\
R_{\vartheta \varphi \vartheta \varphi} & =4 \frac{r^{2}}{f} r_{, u} r_{, \mathrm{v}} \sin ^{2} \vartheta
\end{aligned}
$$

$$
R_{u \vartheta \vee \vartheta}=-r_{, u} r_{, \mathrm{v}}-\frac{f}{4}\left(1-\frac{q^{2}}{r^{2}}\right),
$$

$R_{\mathrm{\vee} \vartheta \vee \vartheta}=-r^{2} \Phi_{, \mathrm{v}}^{2}$

$R_{u \varphi \vee \varphi}=-\left[r_{, u} r_{, \mathrm{v}}+\frac{f}{4}\left(1-\frac{q^{2}}{r^{2}}\right)\right] \sin ^{2} \vartheta$

$R_{u \varphi u \varphi}=-r^{2} \Phi_{, u}^{2} \sin ^{2} \vartheta$

$R_{u \vartheta u \vartheta}=-r^{2} \Phi_{, u}^{2}$,

$R_{\mathrm{V} \varphi \vee \varphi}=-r^{2} \Phi_{, \mathrm{v}}^{2} \sin ^{2} \vartheta$
[1] S.W. Hawking and G.F.R. Ellis, The Large Scale Structure of Space-time (Cambridge University Press, Cambridge, England, 1973).

[2] V.A. Belinsky, I.M. Khalatnikov, and E.M. Lifshitz, Adv. Phys. 19, 525 (1970).

[3] L.M. Burko and A. Ori, in Internal Structure of Black Holes and Spacetime Singularities, edited by L.M. Burko and A. Ori (Institute of Physics, Bristol, 1997).

[4] F.J. Tipler, Phys. Lett. 64A, 8 (1977).

[5] A. Ori, Phys. Rev. Lett. 68, 2117 (1992).

[6] A. Ori, Gen. Relativ. Gravit. 29, 881 (1997).

[7] P.R. Brady, S. Droz, and S.M. Morsink, Phys. Rev. D 58, 084034 (1998).

[8] A. Ori and É.É. Flanagan, Phys. Rev. D 53, R1754 (1996).

[9] A. Ori, Phys. Rev. D 57, 4745 (1998).

[10] W.A. Hiscock, Phys. Lett. 83A, 110 (1981).

[11] E. Poisson and W. Israel, Phys. Rev. D 41, 1796 (1990).

[12] A. Ori, Phys. Rev. Lett. 67, 789 (1991).

[13] P.R. Brady and J.D. Smith, Phys. Rev. Lett. 75, 1256 (1995).

[14] L.M. Burko, Phys. Rev. Lett. 79, 4958 (1997).

[15] L.M. Burko and A. Ori, Phys. Rev. D 57, R7084 (1998).

[16] S. Hod and T. Piran, Phys. Rev. Lett. 81, 1554 (1998); Gen. Relativ. Gravit. 30, 1555 (1998).

[17] This point was stressed by B.C. Nolan, gr-qc/9902020. Nolan tried to present an example of a strong spacelike singularity with a nonsingular $C^{0}$ metric. However, as shown in A. Ori, gr-qc/9902055, Nolan's singularity is, in fact, weak. Thus, the only known examples for strong singularities with nonsingular $C^{0}$ metrics are pathological and unlikely to be realized in nature. In fact, all those singularities are strong only on subsets of measure zero. However, a nonsingular $C^{0}$ metric is not equivalent to the singularity being Tipler weak.

[18] L.M. Burko, Phys. Rev. D 59, 024011 (1999).

[19] R.H. Price, Phys. Rev. D 5, 2419 (1972).

[20] C. Gundlach, R.H. Price, and J. Pullin, Phys. Rev. D 49, 883 (1994).

[21] L.M. Burko and A. Ori, Phys. Rev. D 56, 7820 (1997).

[22] L.M. Burko, Phys. Rev. D 55, 2105 (1997).

[23] C.J.S. Clarke and A. Królak, J. Geom. Phys. 2, 127 (1985).

[24] A. Ori, Phys. Rev. D (in press).

[25] A. Królak, J. Math. Phys. 28, 138 (1987).

[26] R. Herman and W.A. Hiscock, Phys. Rev. D 46, 1863 (1992).

[27] A. Ori, in Internal Structure of Black Holes and Spacetime Singularities [3].

[28] W.A. Hiscock, Phys. Rev. D 15, 3054 (1977).

[29] N.D. Birrell and P.C.W. Davis, Nature (London) 272, 35 (1978).

[30] R. Balbinot and E. Poisson, Phys. Rev. Lett. 70, 13 (1993).

[31] W.G. Anderson, P.R. Brady, W. Israel, and S.M. Morsink, Phys. Rev. Lett. 70, 1041 (1993). 